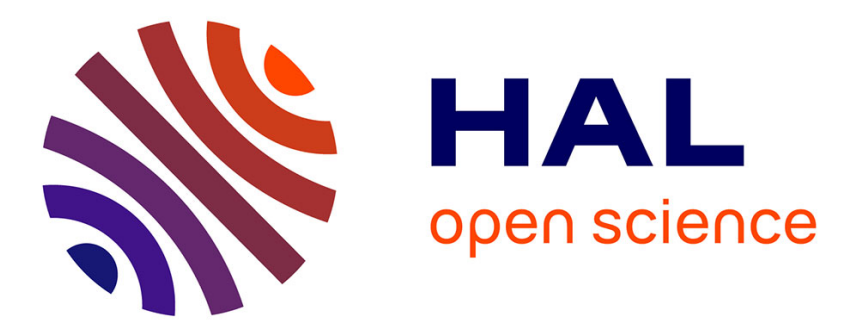

\title{
Fast transform based preconditioners for 2D finite-difference frequency-domain: Waveguides and periodic structures
}

\author{
Alexandre Chabory, B.P. de Hon, A.G. Tijhuis, W.H.A Schilders
}

\section{To cite this version:}

Alexandre Chabory, B.P. de Hon, A.G. Tijhuis, W.H.A Schilders. Fast transform based preconditioners for 2D finite-difference frequency-domain: Waveguides and periodic structures. Journal of Computational Physics, 2008, 227 (16), pp 7755-7767. 10.1016/j.jcp.2008.04.030 • hal-01021585

\section{HAL Id: hal-01021585 \\ https://hal-enac.archives-ouvertes.fr/hal-01021585}

Submitted on 23 Sep 2014

HAL is a multi-disciplinary open access archive for the deposit and dissemination of scientific research documents, whether they are published or not. The documents may come from teaching and research institutions in France or abroad, or from public or private research centers.
L'archive ouverte pluridisciplinaire HAL, est destinée au dépôt et à la diffusion de documents scientifiques de niveau recherche, publiés ou non, émanant des établissements d'enseignement et de recherche français ou étrangers, des laboratoires publics ou privés. 


\title{
Fast Transform Based Preconditioners for 2D Finite-Difference Frequency-Domain - Waveguides and Periodic Structures
}

\author{
A. Chabory ${ }^{a, *, 1}$, B.P. de Hon ${ }^{\text {a }}$, W.H.A. Schilders ${ }^{b}$, \\ A.G. Tijhuis ${ }^{\mathrm{a}}$ \\ ${ }^{a}$ Department of Electrical Engineering, Eindhoven University of Technology, P.O. \\ Box 513, 5600 MB Eindhoven, Netherlands \\ ${ }^{\mathrm{b}}$ Department of Mathematics and Computer Science, Eindhoven University of \\ Technology, P.O. Box 513, 5600 MB Eindhoven, Netherlands
}

\begin{abstract}
The fields scattered by dielectric objects placed inside parallel-plate waveguides and periodic structures in two dimensions may efficiently be computed via a finitedifference frequency-domain (FDFD) method. This involves large, sparse linear systems of equations that may be solved using preconditioned Krylov subspace methods. Our preconditioners involve fast discrete trigonometric transforms and are based on a physical approximation. Simulations show significant gain in terms of computation time and iteration count in comparison with results obtained with preconditioners based on incomplete LU (ILU) factorization. Moreover, with the new preconditioners, the required number of iterations is independent of the grid size.
\end{abstract}

Key words: Finite difference methods, iterative methods, Helmholtz equation, electromagnetic scattering, waveguides, periodic structures. 


\section{Introduction}

Due to the introduction of electromagnetically complex environments, such as metamaterials, in the design of antennas and waveguides and in electromagnetic shielding, the corresponding problems in computational electromagnetics have become more demanding both in size and complexity. Finitedifference methods constitute versatile tools by which these problems may be solved. The most popular of them, the finite-difference time-domain (FDTD) method, initially proposed by Yee [1], leads to an explicit leap-frog scheme for marching on in time. Nevertheless, because it is a time-domain approach, FDTD is in general not suitable for dispersive media, whose properties are frequency-dependent, or for applications where the interest is in the timeharmonic response of the system. In such configurations, one would have to use a frequency-domain method instead, e.g., the finite-difference frequencydomain (FDFD) method. Besides, for many configurations such as 2D waveguides, a frequency-domain approach allows for an exact modal formulation of the absorbing boundary conditions $[2,3]$.

The FDFD approach leads to a sparse linear system $A u=b$. The associated matrix-vector product is fast, which suggests that Krylov subspace iterative methods may constitute suitable solvers. However, due to ill-conditioning, convergence is often slow, so that long computation times are required. One way to speed up the convergence rate is to precondition the system. Instead of

* Corresponding author.

Email address: alexandre.chabory@recherche.enac.fr (A. Chabory).

1 Present adress: ENAC, 7 av. E. Belin, BP 54005, 31055 Toulouse, France. 
the original linear system $A u=b$, a preconditioned system $A_{0}^{-1} A u=A_{0}^{-1} b$ is solved. The matrix $A_{0}$, called the preconditioner, is chosen keeping two criteria in mind: the system $A_{0} w=d$ can be rapidly solved for any vector $d$, and the iterative method converges in less steps with $A_{0}^{-1} A$ than with $A$. The latter is often realized by using an approximated inverse $A_{0}^{-1} \approx A^{-1}$, which improves the conditioning of the system.

A large family of preconditioners already exists for sparse linear systems. Among them, one of the most popular techniques is the incomplete factorization method. The standard incomplete LU factorization (ILU) uses an approximated LU factorization of $A$, skipping parts of the computation by imposing on both $L$ and $U$ the sparsity pattern of $A$. This technique can be modified to allow for extra fill-in during the factorization. Another preconditioning approach widely used in many branches of applied physics is based on multigrid theory. However, in the context of the Helmholtz equation, it has been demonstrated that this leads to difficulties concerning both of the main multigrid components: the smoothing and the coarse grid correction. In [4] and [5], these difficulties are analyzed in detail and measures are proposed to overcome them. Preconditioners that involve fast discrete transforms have been introduced in the preconditioning of Toeplitz matrices by circulant matrices [6]. For the discretized counterpart of the Helmholtz equation, such fast preconditioners have been developed to treat a box-shaped domain filled by a homogeneous medium with absorbing boundary conditions $[7,8]$. For that problem, the application of the preconditioner effectively amounts to replace the boundary conditions by more convenient ones (Dirichlet or Neumann). Larsson and Otto proposed this preconditioning method [3] to treat the propagation of hydro-acoustic waves in a duct. Combined with a domain 
decomposition method, their technique has been extended to domains consisting of layers with different material properties $[9,10]$.

In this article, we consider the FDFD method for two 2D structures containing dielectric scatterers, namely, a parallel-plate waveguide and a transversely periodic structure. The time-harmonic problem is first reduced to the Helmholtz equation and discretized with finite differences. The extent of the computational domain is then limited to the section where the scatterers are located. At the transverse boundaries that limit this section, we use the modal representation of the solution to obtain an exact formulation for the absorbing boundary conditions. To define the preconditioner, we look at the physics of the problem. We notice that the field can be represented as uncoupled propagating or attenuating modes everywhere except in the inhomogeneous regions. Even there, we can expect that an uncoupled modal description constitutes a good approximation. Therefore, we define the preconditioner such that it approximates the linear system by decoupling the mutual influences of all the modal constituents propagating or attenuating inside the structure. The resulting preconditioner can be associated with a matrix with a block structure that is diagonalizable by means of a fast discrete trigonometric transformation. This corresponds to an approximation of the initial 2D problem by several uncoupled 1D problems, associated with an effective dielectric permittivity that is invariant along the transverse coordinate.

We start by presenting the FDFD approach and the preconditioner for the parallel-plate waveguide in Section 2. In Section 3, the same approach is applied to the periodic structure. We discuss a few numerical experiments and 
compare our preconditioner with ILU preconditioners in Section 4.

\section{Parallel-plate waveguides}

\subsection{Configuration and formulation}

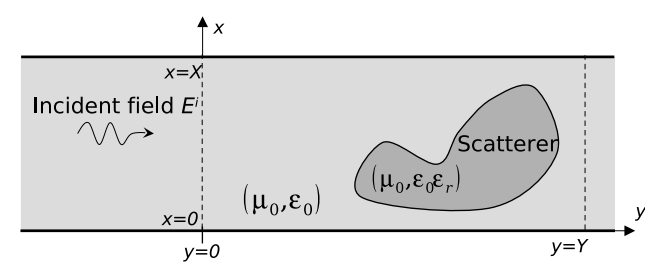

Fig. 1. Parallel-plate waveguide configuration.

In a two dimensional configuration, we consider a parallel-plate waveguide containing dielectric scatterers and excited by an incident TE field, i.e., oriented along $\mathbf{u}_{\mathbf{z}}$, the unit vector in the $z$-direction (Fig. 1). The total field is firstly decomposed into two parts as follows

$$
\mathbf{E}=\left(E_{z}^{i}+E_{z}^{s}\right) \mathbf{u}_{\mathbf{z}}
$$

where $E_{z}^{i}$ and $E_{z}^{s}$ respectively represent the non-vanishing components of the incident and scattered electric fields. In this configuration, the time-harmonic Maxwell equations can be reduced to the Helmholtz equation for $E_{z}^{s}$, i.e.,

$$
\Delta E_{z}^{s}+k_{0}^{2} \varepsilon_{r} E_{z}^{s}=j k_{0} Z_{0} J_{z}^{c}
$$

in which $k_{0}$ and $Z_{0}$ respectively stand for the free-space wavenumber and wave impedance. Further, $j=\sqrt{-1}$ and $\varepsilon_{r}$ is the relative permittivity of the dielectric medium. The contrast current density $J_{z}^{c}$ is defined by

$$
J_{z}^{c}=j \frac{k_{0}}{Z_{0}}\left(\varepsilon_{r}-1\right) E_{z}^{i} .
$$


Furthermore, the following boundary conditions are imposed at the metallic plates

$$
E_{z}^{s}=0 \quad \text { for } x=0 \text { or } x=X .
$$

In the $y$-direction, the physical domain is assumed to be infinite. However, the extent of the computational domain has to remain finite. It is limited to the waveguide section where the scatterers are located by placing two artificial boundaries at $y=0$ and $y=Y$ on which exact discrete absorbing (nonreflecting) boundary conditions will be supplied.

\subsection{Discretization}

We introduce an uniform grid as

$$
\begin{array}{ccc}
x_{m}=m \Delta x & \text { for } m=0, \cdots, M \quad \text { with } \Delta x=X / M, \\
y_{n}=n \Delta y & \text { for } n=0, \cdots, N \quad \text { with } \Delta y=Y / N,
\end{array}
$$

in which $M$ and $N$ are the number of grid points with respect to $x$ and $y$, respectively. The Helmholtz equation (2) is discretized on the interior points of this grid using the central-difference approximation for the spatial derivatives. This leads to

$$
\begin{array}{r}
\frac{1}{(\Delta x)^{2}}\left(E_{z}^{s}[m+1, n]-2 E_{z}^{s}[m, n]+E_{z}^{s}[m-1, n]\right) \\
+\frac{1}{(\Delta y)^{2}}\left(E_{z}^{s}[m, n+1]-2 E_{z}^{s}[m, n]+E_{z}^{s}[m, n-1]\right) \\
+k_{0}^{2} \varepsilon_{r}[m, n] E_{z}^{s}[m, n]=j k_{0} Z_{0} J_{z}^{c}[m, n],
\end{array}
$$

for $m=1, \cdots, M-1$ and $n=1, \cdots, N-1$. 


\subsection{Boundary Conditions}

On the metallic plates, the discretization of (4) gives

$$
E_{z}^{s}[0, n]=E_{z}^{s}[M, n]=0 \quad \text { for } n=0, \cdots, N
$$

For the absorbing boundary conditions, an exact formulation can be obtained from a modal representation of the solution. Our approach is very similar to the ones that lead to nonlocal Dirichlet-to-Neuman maps $[2,3]$, except that we start directly from the discretized problem. Thus, our discretization scheme is rendered self-consistent, which implies that additional discretization errors due to the boundary conditions are avoided. Firstly, we focus on the boundary placed at $y=0$, i.e., $n=0$. The discrete sine transform of the scattered field with respect to $m$ is defined at $n=1$ by

$$
\hat{E}_{z}^{s}[\ell, 1]=\sqrt{\frac{2}{M}} \sum_{m=1}^{M-1} E_{z}^{s}[m, 1] \sin \frac{\pi m \ell}{M}
$$

for $\ell=1, \cdots, M-1$. If we assume that no scatterers are present near the boundary (i.e. $\varepsilon_{r}[m, n]=1$ for $n \leq 1$ ), the scattered field can be represented in terms of modes in this region. An exact absorbing boundary condition is obtained by imposing that each mode should propagate or attenuate away from the computational domain. Hence, $\hat{E}_{z}^{s}[\ell, 0]$ follows from $\hat{E}_{z}^{s}[\ell, 1]$ according to

$$
\hat{E}_{z}^{s}[\ell, 0]=\hat{E}_{z}^{s}[\ell, 1] e^{-\gamma[\ell] \Delta_{y}},
$$

where the propagation coefficients of the modes $\gamma[\ell]$ are obtained directly from the discretized Helmholtz equation, which yields

$$
\gamma[\ell]=\frac{2}{\Delta y} \arcsin \left(\frac{\Delta y}{2} \sqrt{k_{x}^{2}[\ell]-k_{0}^{2}}\right) .
$$


Furthermore, the associated transverse wavenumbers are given by

$$
k_{x}[\ell]=\frac{2}{\Delta x} \sin \frac{\pi \ell}{2 M}
$$

Finally, we perform a backward discrete sine transform to obtain the value of the field on the boundary

$$
E_{z}^{s}[m, 0]=\sqrt{\frac{2}{M}} \sum_{\ell=1}^{M-1} \hat{E}_{z}^{s}[\ell, 0] \sin \frac{\pi m \ell}{M}
$$

From the expressions (8), (9) and (12), we obtain the formulation of the absorbing boundary condition

$$
E_{z}^{s}[., 0]=\left(U \Gamma U^{H}\right) E_{z}^{s}[., 1],
$$

where $U=U^{H}=U^{-1}$ stands for the matrix associated with the discrete sine transform. Its elements are given by

$$
(U)_{\ell, \ell^{\prime}}=\sqrt{\frac{2}{M}} \sin \frac{\pi \ell \ell^{\prime}}{M}, \quad \ell, \ell^{\prime}=1, \cdots M-1
$$

Further,

$$
\Gamma=\operatorname{diag}_{M-1}\left(e^{-\gamma[1] \Delta y}, \cdots, e^{-\gamma[M-1] \Delta y}\right)
$$

is a diagonal matrix describing the propagation and/or attenuation of the modes between $n=1$ and $n=0$. At $y=Y$, we again assume that there are no scatterers beyond or crossing the boundary. A similar analysis leads to

$$
E_{z}^{s}[., N]=\left(U \Gamma U^{H}\right) E_{z}^{s}[., N-1]
$$

\subsection{Linear system}

Using (7), (13) and (16) to account for the field on the boundary of the domain in (6), we obtain a linear system $A u=b$ of size $N_{u}=(M-1)(N-1)$. The 
unknown $u$ and the right-hand-side $b$ are vectors containing the scattered electric field $E_{z}^{s}$ and the source term $j k_{0} Z_{0} J_{z}^{c}$,respectively. The matrix $A$ has a block structure which can be expressed by means of the Kronecker product $($ denoted by $\otimes)[11]$

$$
\begin{aligned}
A= & D_{2 x} \otimes I_{N-1}+I_{M-1} \otimes D_{2 y} \\
& +\frac{1}{(\Delta y)^{2}}\left(U \Gamma U^{H}\right) \otimes\left(B_{1}+B_{N-1}\right)+k_{0}^{2} \Lambda_{\varepsilon_{r}}
\end{aligned}
$$

where $I_{M-1}$ and $I_{N-1}$ denote identity matrices of size $M-1$ and $N-1$, respectively. The matrices $D_{2 x}$ and $D_{2 y}$ are associated with the discretization of the second-order partial derivatives

$$
\begin{aligned}
& D_{2 x}=\frac{1}{(\Delta x)^{2}} \operatorname{tridiag}_{M-1}(1,-2,1), \\
& D_{2 y}=\frac{1}{(\Delta y)^{2}} \operatorname{tridiag}_{N-1}(1,-2,1) .
\end{aligned}
$$

The square matrices $B_{n}$, of size $(N-1)$, are given by

$$
\left(B_{n}\right)_{\ell, \ell^{\prime}}= \begin{cases}1 & \text { for } \ell=\ell^{\prime}=n \\ 0 & \text { otherwise }\end{cases}
$$

Further, $\Lambda_{\varepsilon_{r}}$ is a diagonal matrix containing the relative permittivity values on the interior points of the grid. In the context of iterative solvers based on Krylov subspaces, the computation time needed for one iteration mainly depends on the complexity of the matrix-vector product $A v$ for any $v$. By employing fast algorithms to compute the discrete sine transforms, this complexity is of order $O(M N+M \log M)$, which is very efficient. 


\subsection{Discrete sine transform preconditioner}

Our preconditioner $A_{0}$ is defined so that it decouples all the modal constituents propagating or attenuating inside an effective background. This yields an approximation of the actual configuration only in the inhomogeneous regions where the scatterers are located. In order to examine the mutual influence of these constituents in the original linear system, we analyze the discrete sine transform of $A$ with respect to the transverse direction

$$
\hat{A}=\left(U^{H} \otimes I_{N-1}\right) A\left(U \otimes I_{N-1}\right) .
$$

Using (17) and results about sine transforms of diagonal and tridiagonal matrices [12], we obtain

$$
\begin{aligned}
\hat{A}= & \hat{D}_{2 x} \otimes I_{N-1}+I_{M-1} \otimes D_{2 y} \\
& +\frac{1}{\Delta y^{2}} \Gamma \otimes\left(B_{1}+B_{N-1}\right) \\
& +k_{0}^{2} \sum_{n=1}^{N-1}\left(\hat{T}_{\varepsilon_{r}}^{n}-\hat{H}_{\varepsilon_{r}}^{n}\right) \otimes B_{n},
\end{aligned}
$$

in which $\hat{D}_{2 x}=\operatorname{diag}_{M-1}\left(-k_{x}^{2}[\ell]\right)$. For $n=1, \cdots, N-1, \hat{T}_{\varepsilon_{r}}^{n}$ and $\hat{H}_{\varepsilon_{r}}^{n}$ are the respective Toeplitz and Hankel matrices,

$$
\begin{aligned}
\left(\hat{T}_{\varepsilon_{r}}^{n}\right)_{\ell, \ell^{\prime}} & =\frac{1}{\sqrt{2 M}} \tilde{\varepsilon}_{r}\left[\ell-\ell^{\prime}, n\right], \\
\left(\hat{H}_{\varepsilon_{r}}^{n}\right)_{\ell, \ell^{\prime}} & =\frac{1}{\sqrt{2 M}} \tilde{\varepsilon}_{r}\left[\ell+\ell^{\prime}, n\right],
\end{aligned}
$$

where

$$
\tilde{\varepsilon}_{r}[\ell, n]=\sqrt{\frac{2}{M}} \sum_{m=1}^{M-1} \varepsilon_{r}[m, n] \cos \frac{\pi \ell m}{M} .
$$

Note that $\tilde{\varepsilon}_{r}[\ell, n]$ is the standard discrete cosine transform of the vector

$$
\left(0, \varepsilon_{r}[1, n], \cdots, \varepsilon_{r}[M-1, n], 0\right)^{T}
$$


for each value of $n$. To remove the coupling between the modal constituents in the waveguide, $\hat{A}$ must be approximated by a matrix that is block diagonal. In the context of fast-transform based preconditioners [6], the most commonly used technique is to take the optimal approximation $A_{0}$ of $A$ with respect to the Frobenius norm, i.e., $A_{0}$ is the block diagonal matrix such that $\left\|A-A_{0}\right\|_{F}=$ $\left\|\hat{A}-\hat{A}_{0}\right\|_{F}$ is minimal, where

$$
\left\|A-A_{0}\right\|_{F}=\sum_{\ell, \ell^{\prime}=1}^{N_{u}}\left|(A)_{\ell, \ell^{\prime}}-\left(A_{0}\right)_{\ell, \ell^{\prime}}\right|^{2} .
$$

Because the Frobenius norm remains invariant under unitary transformations, $\hat{A}_{0}$ is obtained upon replacing $\hat{T}_{\varepsilon_{r}}^{n}-\hat{H}_{\varepsilon_{r}}^{n}$ by its diagonal part in (21). It leads to the formulation of the preconditioner

$$
A_{0}=\left(U \otimes I_{N-1}\right) \hat{A}_{0}\left(U^{H} \otimes I_{N-1}\right),
$$

with

$$
\begin{aligned}
\hat{A}_{0}= & \hat{D}_{2 x} \otimes I_{N-1}+I_{M-1} \otimes D_{2 y} \\
& +\frac{1}{(\Delta y)^{2}} \Gamma \otimes\left(B_{1}+B_{N-1}\right) \\
& +k_{0}^{2} \sum_{n=1}^{N-1} \delta\left(\hat{T}_{\varepsilon_{r}}^{n}-\hat{H}_{\varepsilon_{r}}^{n}\right) \otimes B_{n} .
\end{aligned}
$$

$\delta($.$) indicates a diagonal matrix with elements that are the diagonal elements$ of the argument. The decoupling allows for a separate treatment of the modal constituents. Hence, the preconditioner replaces the initial linear system of size $(M-1)(N-1)$ by $M-1$ decoupled systems of size $N-1$. More precisely, to solve the equation $A_{0} w=d$, we compute the sine transform of the right hand side

$$
\hat{d}=\left(U^{H} \otimes I_{N-1}\right) d
$$


Then, we solve the $M-1$ decoupled systems

$$
\hat{A}_{0}^{(\ell)} \hat{w}[\ell, .]=\hat{d}[\ell, .]
$$

where

$$
\begin{aligned}
\hat{A}_{0}^{(\ell)}= & D_{2 y}-k_{x}^{2}[\ell] I_{N-1} \\
& +\frac{e^{-\gamma \ell \ell] \Delta y}}{(\Delta y)^{2}}\left(B_{1}+B_{N-1}\right)+k_{0}{ }^{2} \Lambda_{\varepsilon_{r}^{e}}^{(\ell)},
\end{aligned}
$$

in which $\Lambda_{\varepsilon_{r}^{e}}^{(\ell)}$ are diagonal matrices containing an effective permittivity that depends on the modal order, and on the longitudinal coordinate according to

$$
\varepsilon_{r}^{e}[\ell, n]=\frac{1}{\sqrt{2 M}}\left(\tilde{\varepsilon}_{r}[0, n]-\tilde{\varepsilon}_{r}[2 \ell, n]\right)
$$

Thus, the decoupled systems can be identified with single-mode physical configurations with an effective dielectric permittivity that is invariant along the transverse coordinate. Finally the solution is given by the sine transform of $\hat{w}$

$$
w=\left(U \otimes I_{N-1}\right) \hat{w} .
$$

The use of $A_{0}$ as a preconditioner is only worthwhile if solving $A_{0} w=d$ is inexpensive for any vector $d$. The decoupled systems are tridiagonal and symmetric. Therefore, each one can be solved in $O(N)$ operations. Taking into account the computations of the sine transforms in (28) and (32), the complexity of solving $A_{0} w=d$ is of order $O(M N \log M)$. Hence, during one iteration, the cost of the preconditioner remains moderate, even if it is slightly more expensive than the cost of the matrix-vector product $A v$. 


\section{Periodic structures}

\subsection{Configuration and formulation}

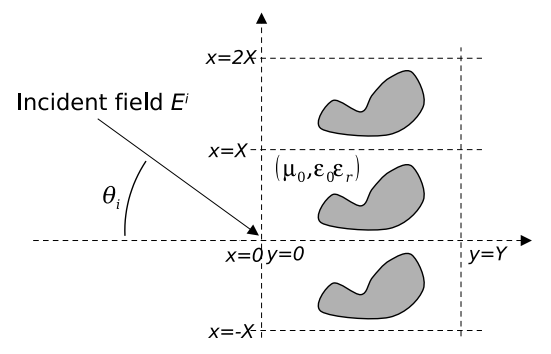

Fig. 2. Periodic structure configuration.

We adopt a similar approach for dielectric structures that are periodic with respect to $x$ (Fig. 2)

$$
\varepsilon_{r}(x+q X, y)=\varepsilon_{r}(x, y), \quad \forall q \in \mathbb{Z}
$$

We assume that the incident field is a TE plane wave

$$
E_{z}^{i}(\mathbf{r})=E_{z 0}^{i} e^{-j \mathbf{k}_{i} \cdot \mathbf{r}}
$$

where $\mathbf{k}_{i}=\left(k_{x i}, k_{y i}\right)^{T}$ stands for the incident wave vector. As in the parallelplate case, the problem can be reduced to the inhomogeneous Helmholtz equation, (2), with a contrast current density source term, as in (3). In the $x$-direction, the extent of the computational domain can be limited to one period $(0 \leq x \leq X)$ by means of Bloch-wave boundary conditions, i.e.,

$$
E_{z}^{s}(X, y)=E_{z}^{s}(0, y) e^{-j k_{x i} X}
$$

In the $y$-direction, the computational domain is restricted to the section where the scatterers are located. 


\subsection{Discretization}

Starting from the grid defined in (5), the discretization of the Helmholtz equation remains as in (6), albeit that $m=0, \cdots, M-1$ and $n=1, \cdots, N-1$, i.e., $m=0$ is now included. The discretized equation, combined with modal absorbing and Bloch-wave boundary conditions, yield a linear system $A u=b$ of size $M(N-1)$,

$$
\begin{aligned}
A= & D_{2 x} \otimes I_{N-1}+I_{M} \otimes D_{2 y} \\
& +\frac{1}{(\Delta y)^{2}}\left(U \Gamma U^{H}\right) \otimes\left(B_{1}+B_{N-1}\right)+k_{0}^{2} \Lambda_{\varepsilon_{r}} .
\end{aligned}
$$

Note that (36) has the same form as (17), albeit that there are some slight differences in the definition of the constituent terms. The diagonal matrix $\Lambda_{\varepsilon_{r}}$ contains the relative permittivity values both on the interior points and on the boundary $m=0$. In view of Bloch-wave boundary conditions, the matrix $D_{2 x}$ becomes

$$
D_{2 x}=\frac{1}{(\Delta x)^{2}}\left[\operatorname{tridiag}_{M}(1,-2,1)+P\right]
$$

where

$$
(P)_{\ell+1, \ell^{\prime}+1}= \begin{cases}e^{j k_{x i} X} & \text { for } \ell=0, \ell^{\prime}=M-1 \\ e^{-j k_{x i} X} & \text { for } \ell=M-1, \ell^{\prime}=0 \\ 0 & \text { otherwise. }\end{cases}
$$

The unitary transformation $U$ is associated with the modal representation of the solution in the periodic structure. It may be expressed as

$$
U^{H}=F \Lambda_{i}
$$

where $\Lambda_{i}$ introduces the phase shifts associated with the angle of incidence,

$$
\Lambda_{i}=\operatorname{diag}_{M}\left(1, e^{j k_{x i} \Delta x}, \cdots, e^{j(M-1) k_{x i} \Delta_{x}}\right)
$$


and $F$ is the matrix associated with the discrete Fourier transform of size $M$. Its elements are given by

$$
(F)_{\ell+1, \ell^{\prime}+1}=\frac{e^{j 2 \pi \ell \ell^{\prime} / M}}{\sqrt{M}}, \quad \ell, \ell^{\prime}=0, \cdots M-1 .
$$

Further, the diagonal matrix $\Gamma$ contains the propagation and/or attenuation factors of modes that have traveled a distance $\Delta y$, i.e.,

$$
\Gamma=\operatorname{diag}_{M}\left(e^{-\gamma[0] \Delta y}, \cdots, e^{-\gamma[M-1] \Delta y}\right)
$$

The propagation coefficients $\gamma$ are associated with the discretized Helmholtz equation with Bloch-wave boundary conditions,

$$
\gamma[\ell]=\frac{2}{\Delta y} \arcsin \left(\frac{\Delta y}{2} \sqrt{k_{x}^{2}[\ell]-k_{0}^{2}}\right)
$$

where

$$
k_{x}[\ell]=\frac{2}{\Delta x} \sin \left(\frac{k_{x i} \Delta x}{2}+\frac{\pi \ell}{M}\right)
$$

are the corresponding transverse wavenumbers.

\subsection{Preconditioner}

We start with the formulation of the $U$-transform of $A$

$$
\hat{A}=\left(U^{H} \otimes I_{N-1}\right) A\left(U \otimes I_{N-1}\right) .
$$

Using (36) and results from [6], we obtain an explicit expression for $\hat{A}$

$$
\begin{aligned}
\hat{A}= & \hat{D}_{2 x} \otimes I_{N-1}+I_{M} \otimes D_{2 y} \\
& +\frac{1}{\Delta_{y}^{2}} \Gamma \otimes\left(B_{1}+B_{N-1}\right) \\
& +\sum_{n=1}^{N-1} \hat{C}_{\varepsilon_{r}}^{n} \otimes B_{n}
\end{aligned}
$$


where the circulant matrices $\hat{C}_{\varepsilon_{r}}^{n}$ are generated by the discrete Fourier transform $\breve{\varepsilon}_{r}[\ell, n]$ of $\varepsilon_{r}[m, n]$ with respect to $m$. Their elements are given by

$$
\left(\hat{C}_{\varepsilon_{r}}^{n}\right)_{\ell, \ell^{\prime}}=\frac{1}{\sqrt{M}} \breve{\varepsilon}_{r}\left[\ell-\ell^{\prime}, n\right]
$$

The preconditioner $A_{0}$ is defined as the optimal approximation of $A$ with respect to the Frobenius norm such that the modal constituents are decoupled. In the periodic case, this amounts to

$$
A_{0}=\left(U \otimes I_{N-1}\right) \hat{A}_{0}\left(U^{H} \otimes I_{N-1}\right)
$$

with

$$
\begin{aligned}
\hat{A}_{0}= & \hat{D}_{2 x} \otimes I_{N-1}+I_{M} \otimes D_{2 y} \\
& +\frac{1}{(\Delta y)^{2}} \Gamma \otimes\left(B_{1}+B_{N-1}\right) \\
& +\sum_{n=1}^{N-1} \delta\left(\hat{C}_{\varepsilon_{r}}^{n}\right) \otimes B_{n} .
\end{aligned}
$$

To solve $A_{0} w=d, d$ is subjected to a unitary transformation. Then the decoupled systems $\hat{A}_{0}^{(\ell)} \hat{w}[\ell,]=.\hat{d}[\ell,$.$] are solved for \ell=0, \cdots, M-1$, where

$$
\begin{aligned}
\hat{A}_{0}^{(\ell)}= & D_{2 y}-k_{x}^{2}[\ell] I_{N-1} \\
& +\frac{e^{-\gamma[\ell] \Delta y}}{(\Delta y)^{2}}\left(B_{1}+B_{N-1}\right)+k_{0}^{2} \Lambda_{\varepsilon_{r}^{e}},
\end{aligned}
$$

in which $\Lambda_{\varepsilon_{r}^{e}}$ is a diagonal matrix, with the effective permittivity

$$
\varepsilon_{r}^{e}[\ell, n]=\frac{1}{\sqrt{M}} \breve{\varepsilon}_{r}[0, n]=\frac{1}{M} \sum_{m=0}^{M-1} \varepsilon_{r}[m, n]
$$

on its diagonal. Just as in the parallel-plate waveguide case, the decoupled systems can be interpreted as single-mode configurations with an effective permittivity independent of the transverse coordinate. However, the result is slightly different because the discrete transform we use is not the same. Here, the effective permittivity is also independent of the mode order and 
corresponds to the mean value of $\varepsilon_{r}$ with respect to $m$.

Finally, $w$ is obtained via a unitary transformation on $\hat{w}$.

\section{Numerical experiments}

\subsection{Parallel-plate waveguide}

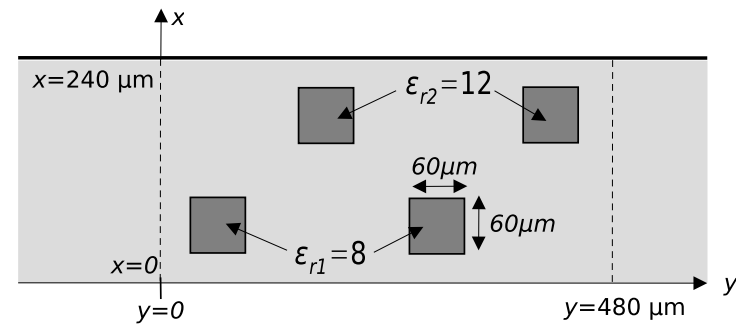

Fig. 3. Geometry of the waveguide filled by 4 scatterers.

As an illustration, we consider a parallel-plate waveguide section filled by 4 square dielectric scatterers of relative permittivity $\varepsilon_{r 1}=8$ and $\varepsilon_{r 2}=12$ (Fig. 3). The excitation is provided by a first-order incident mode at a frequency of $1000 \mathrm{GHz}$. The iterative method we employ is BiCGstab(2) for which each iteration involves the computation of 4 matrix-vector products. This choice is motivated by the numerical experiments conducted in [13], in which for 5 different sparse linear systems, shorter computation times are obtained with BiCGstab(2) than with other iterative methods (Bi-CG, CGS, GMRES, BiCGstab(l) with $l \neq 2$ ). In all the simulations, the stopping criterion is defined such that the norm of the residual, $r_{i}=b-A u_{i}$, after the last iteration is reduced by a factor of $10^{-6}$ with respect to the norm of $b$. The number of points is determined so that the grid steps along $x$ and $y$ are the same, and that the criterion $k_{0} \sqrt{\varepsilon_{r}} \Delta x<\pi / 5$ (i.e. at least ten points per local wavelength) is satisfied everywhere. The choice $N=2 M=128$ fulfills that 
criteria.

In Fig. 4, we have depicted the amplitude of the scattered field computed

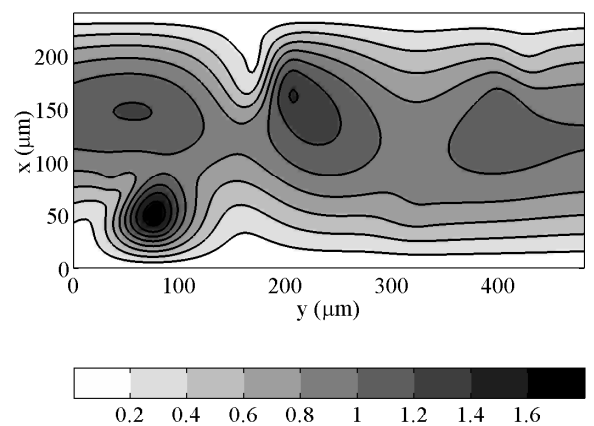

Fig. 4. Amplitude of the electric field $E_{z}^{s}$.

with these parameters. To evaluate the efficiency of the preconditioner in this simulation, we compare in Table 1 the number of matrix-vector products and the computation times obtained for different scenarios, viz., without any preconditioner, with ILU(0) and ILU(3) factorizations, and with the fast transform based preconditioner (FTP). Observe that FTP outperforms ILU(0) and ILU(3). The decrease in the computation time is about a factor of 9.5 as compared with no preconditioning, about a factor of 3 with ILU(0), and about a factor of 2 with $\operatorname{ILU}(3)$.

Let us investigate which parameters play a role in the performance of the

\begin{tabular}{l|cccc} 
& No & $\operatorname{ILU}(0)$ & $\operatorname{ILU}(3)$ & FTP \\
\hline Matrix-vector products & 3357 & 429 & 133 & 65 \\
CPU time (s) & 2.18 & 0.71 & 0.45 & 0.23
\end{tabular}

Table 1

Number of matrix-vector products and computation times without any preconditioner, with ILU(0), with ILU(3) and with FTP.

preconditioner. In Fig. 5, we keep the same physical configuration but we modify the grid size. Observe that the FTP preconditioner yields almost the 
same number of iterations regardless of the grid size. By contrast, if no preconditioner is used or an ILU preconditioner, then the number of iterations increases significantly when the grid size is decreased.

In the next set of simulations, the influence of two physical parameters is
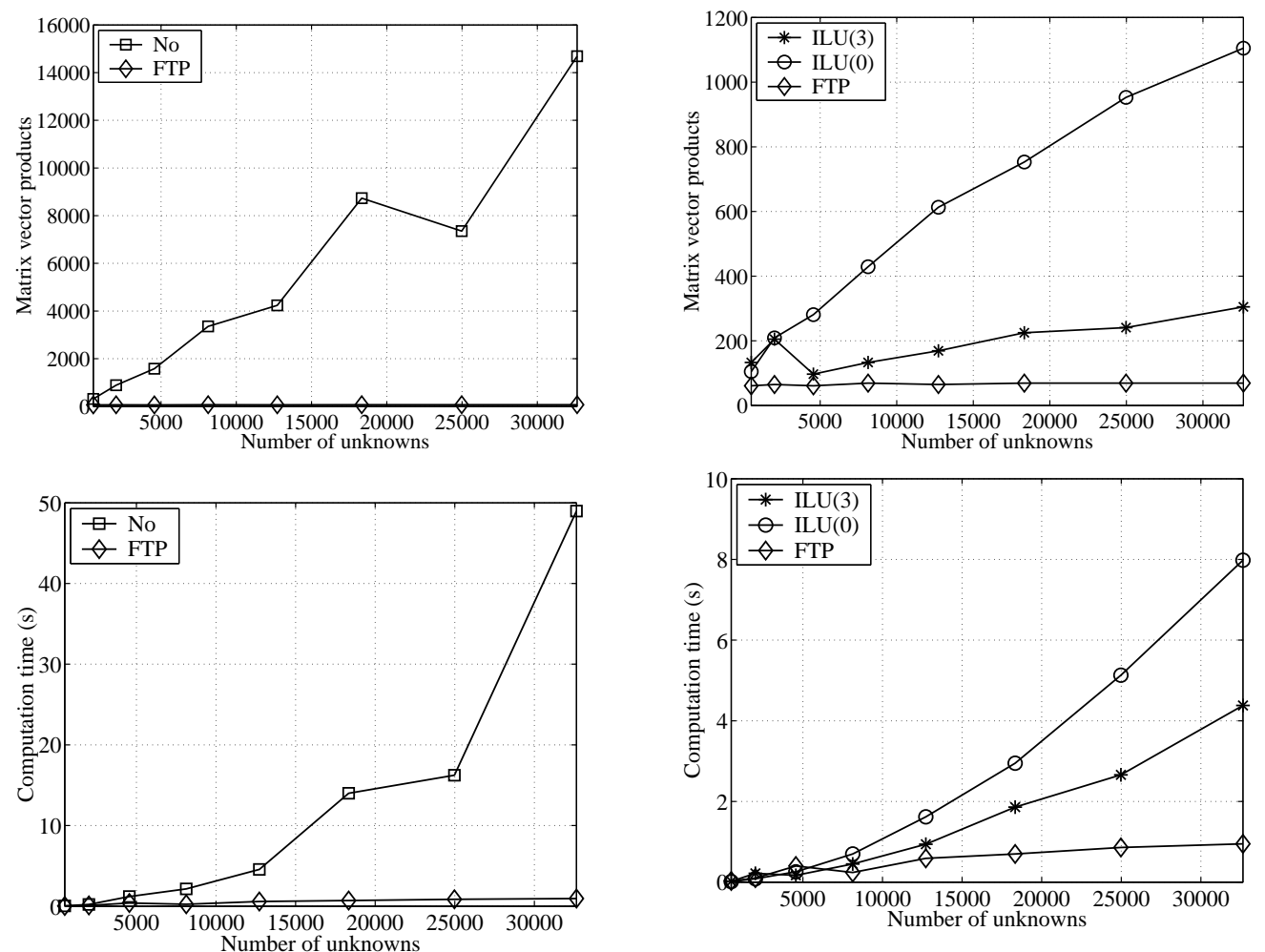

(a)

(b)

Fig. 5. Influence of the grid size on the performances of the preconditioners: (a) Comparison with no preconditioner, (b) Comparison with ILU(0) and ILU(3).

analyzed. In Fig. 6, we show that FTP outperforms ILU(0) and ILU(3) in terms of the iteration count for relative permittivity values $\varepsilon_{r 2}=12$ and $\varepsilon_{r 1}$ varying from 2 to 30 . Besides, we observe that high permittivity contrasts require more iterations with all methods. For FTP, this behavior can be related to the decoupling approximation. When the dielectric profile has strong transversal variations, the condition $\left|\tilde{\varepsilon}_{r}[0, n]\right| \gg\left|\tilde{\varepsilon}_{r}[l, n]\right|$ for $l \neq 0$ does not hold. Therefore, the diagonal terms in $\hat{T}_{\varepsilon_{r}}^{n}-\hat{H}_{\varepsilon_{r}}^{n}$ cease to be dominant, which 
affects the conditioning of $A_{0}^{-1} A$, and yields a degradation of the performance. In Fig. 6, also the influence of the frequency on the preconditioners is inves-
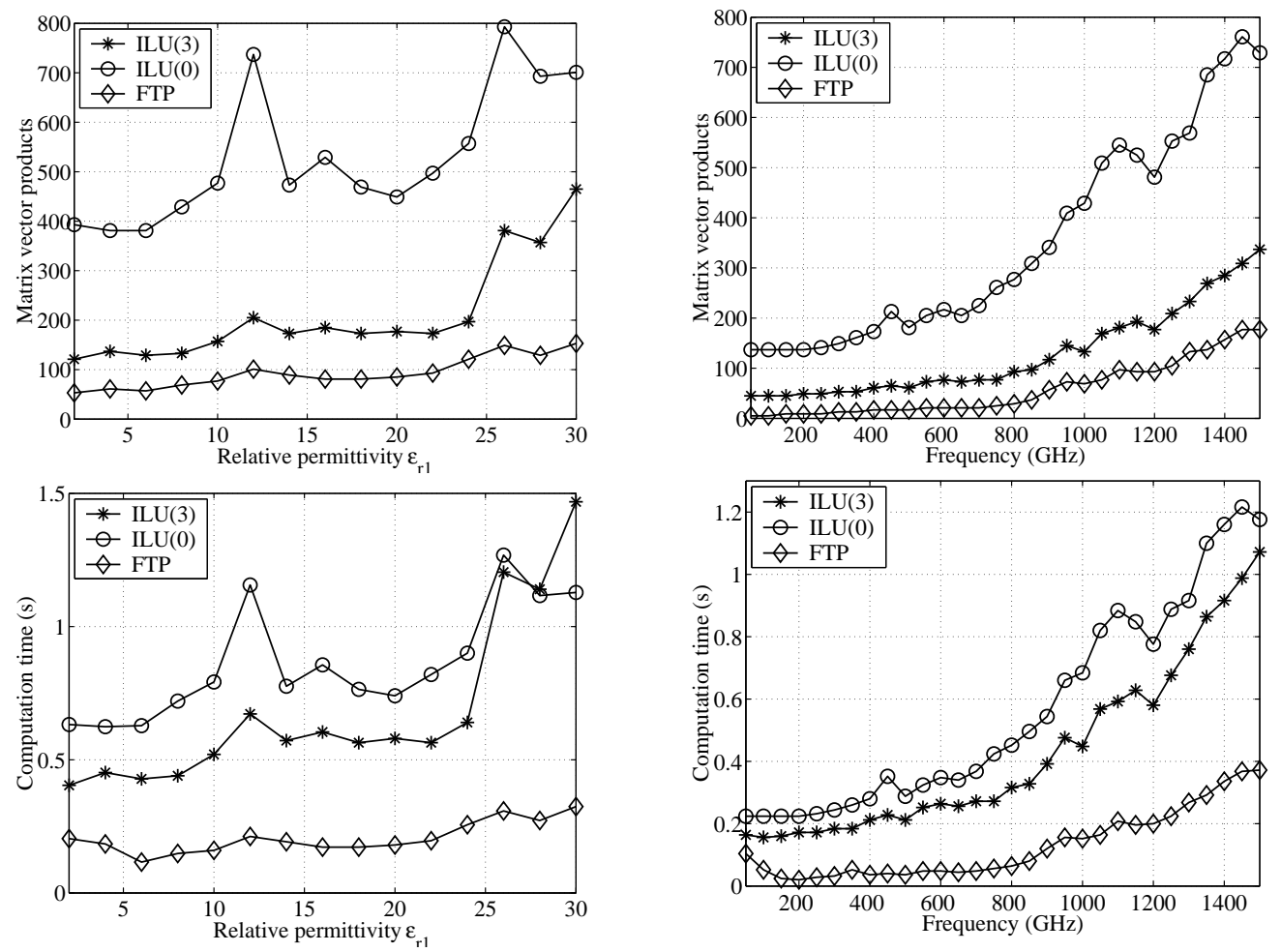

(a)

(b)

Fig. 6. Influence of 2 physical parameters on the performances of the preconditioners:

(a) The relative permittivity $\varepsilon_{r 1}$, (b) The frequency.

tigated. Even if FTP comprises a considerable improvement over ILU(0) and ILU(3) in the entire frequency range, we observe a significant degradation of the performance at high frequencies.

In the last simulation of this section, we look at the performances of the preconditioners when the limits of the scatterers are not parallel to one of the axes. In Table 2, we show the results obtained when the four scatterers are rotated an angle of $45^{\circ}$. Note that again FTP outperforms ILU(0) and ILU(3) in terms of iteration count and computation time. 


\begin{tabular}{l|cccc} 
& No & ILU(0) & ILU(3) & FTP \\
\hline Matrix-vector products & 1393 & 313 & 101 & 45 \\
CPU time (s) & 0.94 & 0.52 & 0.35 & 0.19
\end{tabular}

Table 2

Performance of the preconditioners when the scatterers are rotated an angle of $45^{\circ}$.

\subsection{Periodic structure}

Upon imposing Bloch-wave boundary conditions at $x=0$ and $x=X$, we obtain an electromagnetic-band-gap (EBG) crystal working in the millimeterwave range. This kind of structure is generally electromagnetically characterized by its transmission and reflection coefficients in the specular directions. We have examined the ability of the FTP-preconditioned FDFD to obtain these coefficients for a wide range of frequencies and angles of incidence. In Fig. 7a, the simulation, performed at normal incidence and up to $1400 \mathrm{GHz}$, confirms the presence of a band gap centered at $700 \mathrm{GHz}$ and a bandwidth of $400 \mathrm{GHz}$. However, as in the waveguide case, the performance of our method deteriorates at high frequencies. The influence of the angle of incidence is shown at a frequency of $1000 \mathrm{GHz}$ in Fig. 7b. No significant effects are noticed in the iteration count, even at grazing incidence. For validation purposes, we have checked that exactly the same results are obtained when the computation domain includes two periods of the structure. 

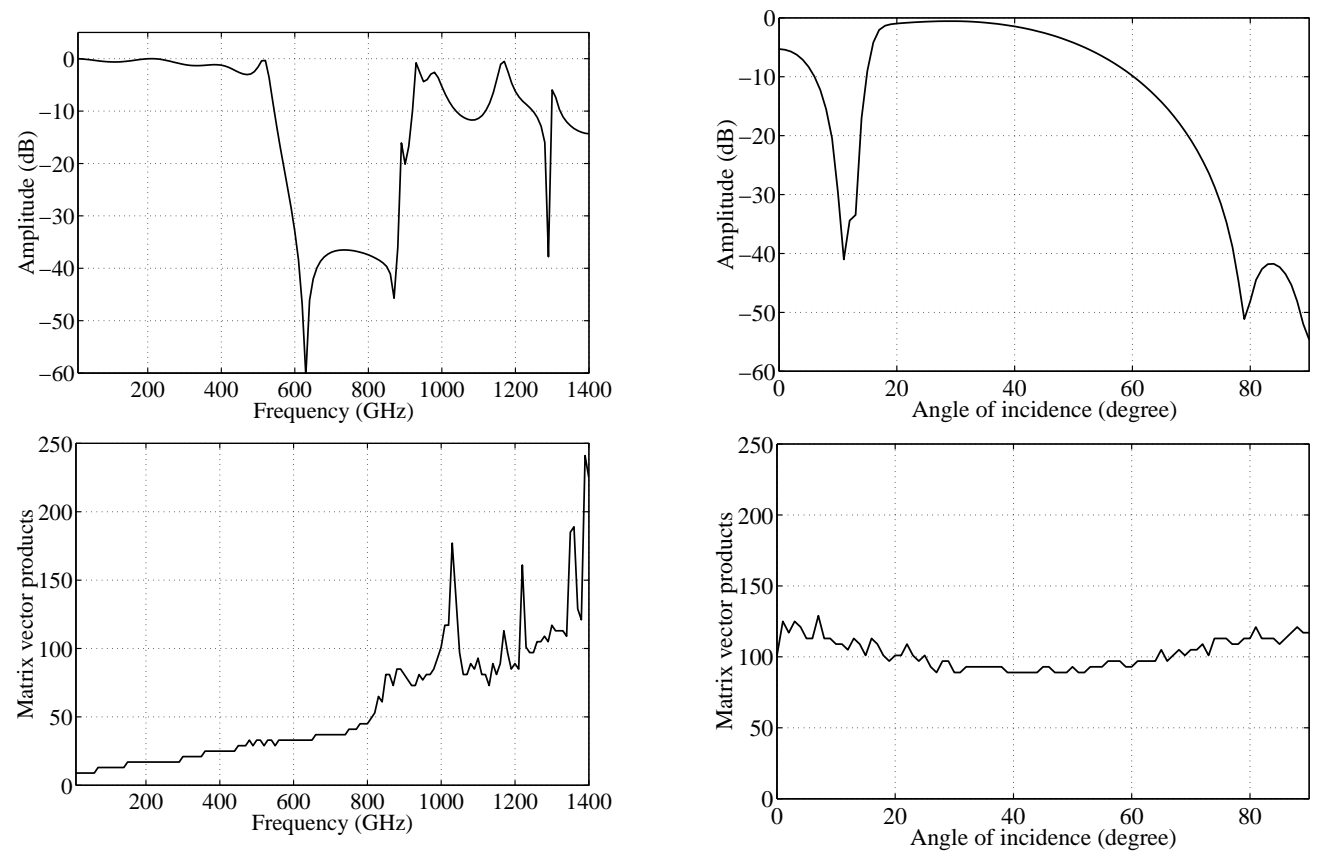

(a)

(b)

Fig. 7. Evolution of the transmission coefficient with respect to: (a) The frequency at normal incidence. (b) The incidence angle at 1000GHz.

\section{Conclusion}

We have proposed an FDFD method to compute the fields scattered by dielectric objects placed inside a parallel-plate waveguide or arranged periodically with respect to the transverse direction. We have used the modal representation of the solution to obtain an exact discretized formulation for the absorbing boundary conditions. The main result is the development of preconditioners by which a significant acceleration may be achieved. These fast preconditioners are based on a physical approximation and involve discrete fast transforms. They are developed so that they introduce approximations only in the inhomogeneous sections of the structure. They are defined as optimal approximations with respect to the Frobenius norm, such that the modal constituents in the structure are decoupled. They amount to replacing the original $2 \mathrm{D}$ con- 
figuration by several 1D decoupled effective configurations. Numerical tests have shown that our approach outperforms significantly ILU(0) and ILU(3) preconditioners in terms of the computation time. Furthermore, we have observed that although the iteration count increases with the permittivity contrast and with frequency, it is independent of the grid size. We have also shown that this method is suitable to compute the transmission coefficients of 2D electromagnetic-band-gap crystals. An important extension of this work would be to solve three-dimensional electromagnetic problems for which the complete Yee staggered grid for vector fields is needed.

\section{Acknowledgment}

The authors would like to thank the Dutch Organization for Scientific Research (NWO) for supporting this work under contract 99008541.

\section{References}

[1] K.S. Yee, Numerical solution of initial boundary value problems involving Maxwell's equations in isotropic media, IEEE Trans. Antennas Propagat., 14, 3, pp. 302-307 (1966).

[2] G.J. Fix, S.P. Marin, "Variational methods for underwater acoustic problems", Journal of Computational Physics, 28, pp. 253-270 (1978).

[3] K. Otto, E. Larsson, "Iterative solution of the Helmholtz equation by a secondorder method", SIAM J. Matrix Anal. Appl., 21, 1, pp. 209-229 (1999).

[4] H.C. Elman, O.G. Ernst, D.P. O'Leary, "A multigrid method enhanced by 
Krylov subspace iteration for discrete Helmholtz equations", SIAM J. Sci. Comput., 23, 4, pp. 1290-1314 (2001).

[5] Y.A. Erlangga, C.W. Oosterlee, C. Vuik, "A novel multigrid based preconditioner for heterogeneous Helmholtz problems", SIAM J. Sci. Comput., 27, 4, pp. 1471-1492 (2006).

[6] R.H. Chan, M.K. Ng, "Conjugate gradient methods for Toeplitz systems, SIAM Review, 38, 3, pp. 427-482 (1996).

[7] H.C. Elman, P.O. O'Leary, "Efficient iterative solution of the three dimensional Helmholtz equation", Journal of Computational Physics, 142, pp. 163-181 (1998).

[8] H.C. Elman, P.O. O'Leary, Eigenanalysis of some preconditioned Helmholtz problems, Num. Math., 83, 2, pp. 231-257 (1999).

[9] E. Larsson, "A domain decomposition method for the Helmholtz equation in a multilayer domain", SIAM J. Sci. Comput., 20, 5 , pp. 1713-1731 (1999).

[10] E. Larsson, S. Holmgren, "Parallel solution of the Helmholtz equation in a multilayer domain", BIT Numerical Mathematics, 43, 2, pp. 387-411 (2003).

[11] Alexander Graham, Kronecker products and matrix calculus with applications, Ellis Horwood (Chichester, 1981).

[12] T. Huckle, Fast transform for tridiagonal linear equations, BIT Numerical Mathematics, 34, 1, pp. 99-112 (1994).

[13] G.L.G. Sleijpen, D.R. Fokkema, "BiCGstab(l) for linear equations involving unsymmetric matrices with complex spectrum", Electronic Transactions on Numerical Analysis, 1, pp. 11-32 (1993). 